\title{
Stanniocalcin 1 is a prognostic biomarker in glioma
}

\author{
WEIJIAN LUO, DONG CHEN, HAO WANG and JILIANG HU \\ Department of Neurosurgery, Shenzhen People's Hospital, Second Clinical Medical \\ College of Ji'nan University, Shenzhen, Guangdong 518020, P.R. China
}

Received October 4, 2019; Accepted April 1, 2020

DOI: $10.3892 / \mathrm{ol} .2020 .11792$

\begin{abstract}
Malignant gliomas are the most common type of primary malignancy of the central nervous system with a poor prognosis. Stanniocalcin 1 (STC1) is closely associated with tumor genesis and development. However, its role in the development and progression of glioma is poorly understood. In silico analysis, The Cancer Genome Atlas (TCGA), Chinese Glioma Genome Atlas (CGGA), Rembrandt and GSE16011 datasets were used to assess the expression levels of STC1 in non-tumor brain tissues and gliomas. Moreover, reverse transcription-quantitative PCR and immunohistochemistry were used to detect STC1 expression in tumor tissues collected in the Department of Neurosurgery of Shenzhen People's Hospital (Shenzhen, China). The association between STC1 expression and different molecular pathological features was analyzed in four public datasets, as well as via Kaplan-Meier analysis. Furthermore, normalized mRNA expression in TCGA was used to perform Gene Ontology analysis. It was revealed that STC1 expression was significantly elevated in glioma tissues compared with the non-tumor brain tissues, both in silico analysis and via cohort validation. According to TCGA, CGGA, Rembrandt and GSE16011 datasets, it was identified that STC1 expression was increased in high grade glioma compared with low grade glioma. In addition, the results indicated STC1 expression was enriched in the isocitrate dehydrogenase (IDH) wild-type and mesenchymal subtype in TCGA, GSE16011 and Rembrandt datasets. Moreover, it was demonstrated that patients with higher STC1 expression exhibited shorter overall survival times compared with those with lower STC1 expression using Kaplan-Meier analysis, according to both the public datasets and validation cohort. Furthermore, the results of the Gene Ontology analysis demonstrated that STC1 was primarily involved in the reorganization of extracellular matrix and was significantly
\end{abstract}

Correspondence to: Professor Jiliang Hu, Department of Neurosurgery, Shenzhen People's Hospital, Second Clinical Medical College of Ji'nan University, 1017 Dongmen North Road, Shenzhen, Guangdong 518020, P.R. China

E-mail: nankeyimeng6e@sina.com

Key words: stanniocalcin 1, bioinformatics analysis, glioma, prognosis, biomarker correlated with invasive-related proteins. Therefore, the present results indicate that STC1 was upregulated in glioma tissues and may represent a prognostic biomarker in patients with glioma.

\section{Introduction}

Glioma is the most common and most malignant tumor type in the brain and accounts for $\sim 80 \%$ of primary malignant brain tumors (1). Currently, the treatment of glioma primarily comprises surgical resection combined with postoperative radiotherapy and chemotherapy (2). However, the median overall survival time is 14 months and the 5-year survival rate of patients with glioma is $<10 \%$, which is not satisfactory (3). Previous studies have reported that glioma is caused by genetic alterations and genetic accumulations $(4,5)$. Moreover, the malignant process of glioma is regulated by a complex gene network comprising of genes, such as p53 and pTEN (6). Therefore, it is essential to identify molecules that may serve a key role in the regulation of the malignant process of glioma.

Stanniocalcin 1 (STC1) is a glycoprotein originally identified in 1996, and is reported to influence the homeostasis of calcium and phosphate (7). Furthermore, STC1 is expressed in several tissue types, including the brain, thymus, spleen, colon and ovaries, and regulates numerous physiological and pathological functions, such as hypoxia, tumorigenesis, angiogenesis and cell proliferation $(6,8,9)$. In addition, STC1 can be secreted into the peripheral blood or body fluid by cells (10). Several studies have reported that circulating STC1 can be used as a promising serum candidate biomarker for tracking the progression of several diseases (11-13). It has also been revealed that the expression of STC1 is significantly correlated with TNM stage in patients with clear cell renal carcinoma (9). Moreover, STC1 is upregulated in ovarian cancer tissue, and may influence ovarian tumorigenesis (12). STC1 expression has been previously detected in a small patient cohort that contained 60 glioma tissues, and it was reported that STC1 is upregulated as glioma grade increases and is correlated with the prognosis of patients with glioma (14). Furthermore, overexpression of STC1 in U87 and LN-229 cells can enhance stem-like traits via regulating Neurogenic locus notch homolog protein 1 (NOTCH) signaling (15). However, the oncogenic role of STC1 in glioma requires further investigation.

The present study aimed to investigate the expression pattern of STC1 in glioma and its association with glioma 
grade, molecular subtypes and clinical prognosis. In addition, Gene Ontology (GO) analysis was performed to understand the potential oncogenic role of STC1 in glioma.

\section{Materials and methods}

Patient samples. This study was carried out on 80 glioma tissues that were collected from the Department of Neurosurgery of Shenzhen People's Hospital from April 2013 to July 2016 (Shenzen, China). The patient cohort consisted of 51 females and 29 males with an average age of 47.54 years old and an age range of 14-72 years old. Patients were followed-up every 3 months for the first 2 years post-surgery, every 6 months for the following 3 years and finally every 12 months for the next 5 years. The primary end point was overall survival time (OS). An additional 10 non-tumor tissues were collected from patients with traumatic brain injury or hypertensive intracerebral hemorrhage. The non-tumor group was comprised of 4 females and 6 males with an average age of 45.2 years old and an age range of 33-68 years old. Sections of the tissues were used for paraffin-embedding once the tumor samples were collected and others were stored at $-80^{\circ} \mathrm{C}$ for further analysis. None of the involved patients received radiotherapy or chemotherapy prior to surgery. The present study was approved by the Ethics Committee of Shenzhen People's Hospital. All patients included in this study provided signed informed consent.

Bioinformatics analysis. Normalized RNAseq data and corresponding clinical material from The Cancer Genome Atlas (TCGA), Rembrandt datasets and GSE16011 were all downloaded from Gliovis (http://gliovis.bioinfo.cnio. es/) (16). TCGA is a public database (http://cancergenome. nih.gov/) that includes 29 cancer types, along with related gene expression and clinical information. Rembrandt dataset (https://caintegrator.nci.nih.gov/rembrandt) is based on 524 Affymetrix U133 2.0 plus microarrays and contained 228 GBM samples and 143 lower grade glioma (WHO II-III) samples. GSE16011 is a public dataset that contains 276 glioma samples of all histology and 8 control samples (17). $\mathrm{GO}$ analysis was also performed in the platform of Gliovis. Through screening comparison, H-cluster analysis was used to analyze the expression of differential expressed genes (DEGS) and functional enrichment was studied by GO analysis. In addition, mRNAseq 693 dataset consisting of 693 glioma tissues with different grades (WHO I-IV) was downloaded from the Chinese Glioma Genome Atlas (CGGA) (18). Low grade glioma (LGG) was defined as WHO grade I-II and high grade glioma (HGG) was defined as WHO grade III-IV according to the 2016 WHO classification of central nervous system tumors (19). According to the WHO 2016 criteria, adult diffuse glioma centers around isocitrate dehydrogenase (IDH) (19). Molecular subtypes in public datasets were defined as described before (20).

Immunohistochemical (IHC) staining. Paraffin-embedded glioma tissues and control brain tissues were used for IHC analysis. Glioma tissues were dewaxed, hydrated and incubated with $3 \%$ hydrogen peroxide for $10 \mathrm{~min}$. The $4 \mathrm{~mm}$ thick sections were then washed three times with PBS for $5 \mathrm{~min}$ each time. Slides were boiled in $0.01 \mathrm{M}$ sodium citrate buffer $(\mathrm{pH}=6.0)$ for $10 \mathrm{~min}$ using a microwave and then allowed to cool for an additional $20 \mathrm{~min}$. Subsequently, 1\% BSA (Beijing Solarbio Science \& Technology Co., Ltd.) was used to block non-specific staining at room temperature for $30 \mathrm{~min}$. Sections were then incubated with STC1 rabbit monoclonal antibody (1:1,000; cat. no. 20621-AP; ProteinTech Group, Inc.) overnight at $4^{\circ} \mathrm{C}$. The next day, sections were incubated with horseradish peroxidase (HRP) conjugated-secondary antibody (1:10,000; cat. no. ANT020; Antgene, Wuhan Antejie Biotechnology Co., Ltd.) for $1 \mathrm{~h}$ at room temperature. Neutral resin was used to seal the slices which were left to dry naturally. After staining, two experienced pathologists were responsible for the evaluation of section staining under an automatic microscope (Olympus BX51; Olympus Corporation), which was performed independently. Images were taken under the microscope at $\mathrm{x} 200 \mathrm{x}$ and x400 magnifications. If the two observers disagreed, a third reader reviewed the images and the final score was given by consensus. IHC score was calculated according to the positive rate of cell staining (number of positively-stained cells per 100 cells) and staining intensity. The staining positive rate was defined as follows: $<5 \%, 0$ points; $5-25 \%, 1$ point; $26-50 \%$, 2 points; $51-75 \%$ score, 3 points; and $>75 \%$ score, 4 points. In addition, staining intensity was scored manually as follows: 0 point, non-stained; 1 point, light yellow; 2 points, brown; and 3 points, dark brown. Final quantization was obtained by multiplying the two scores. The overall score was defined as: 0 , negative; 1-4, weak; 5-8, positive; and 9-12, strong. An IHC score $\leq 5$ was defined as low STC 1 expression and $>5$ points was defined as high STC1 expression.

$R N A$ isolation and reverse transcription-quantitative PCR $(R T-q P C R)$. TRIzol ${ }^{\circledR}$ reagent (Invitrogen; Thermo Fisher Scientific, Inc.) was used to extract total RNA from glioma tissues. PrimeScript RT reagent kit with gDNA Eraser (Takara Bio, Inc.) was used to prepare cDNA and SYBR Green II mixture (Takara Bio, Inc.) was used for RT-qPCR. The thermocycling conditions were as follows: $95^{\circ} \mathrm{C}$ for $15 \mathrm{sec}$ followed by 40 cycles of $60^{\circ} \mathrm{C}$ for $30 \mathrm{sec}$. GAPDH was used as the reference gene. All the operations were performed according to the manufacturer's protocols. The specific primer pairs used in this study were as follows: STC1 forward, 5'-ATC ACATTCCAGCAGGCTTC-3' and reverse, 5'-CCTGAAGCC ATCACTGAGGT-3'; and GAPDH forward, 5'-AACTAGACG ATCACAGCGATGA-3' and reverse, 5'-ACTATCGCAGAC GGACTAC-3'. The quantification of relative expression used $2^{-\Delta \Delta C q}$ method described previously (21).

Statistical analysis. Continuous variables are presented as the mean \pm SD. Comparisons between the two groups were performed using independent sample unpaired t-tests and one-way ANOVA with post hoc Tukey test was used for comparing $\geq 3$ groups . Kaplan-Meier with Log-Rank test was used to analyze the association between STC1 expression and survival time in patients with glioma; 50\% of STC1 expression was used as a cutoff point in Kaplan-Meier analysis. Correlation analysis between STC1 expression and invasion-related markers was performed using Pearson correlation. Comparisons of categorical variables between the groups were performed using the Fishers exact $\chi^{2}$ test. Univariate 

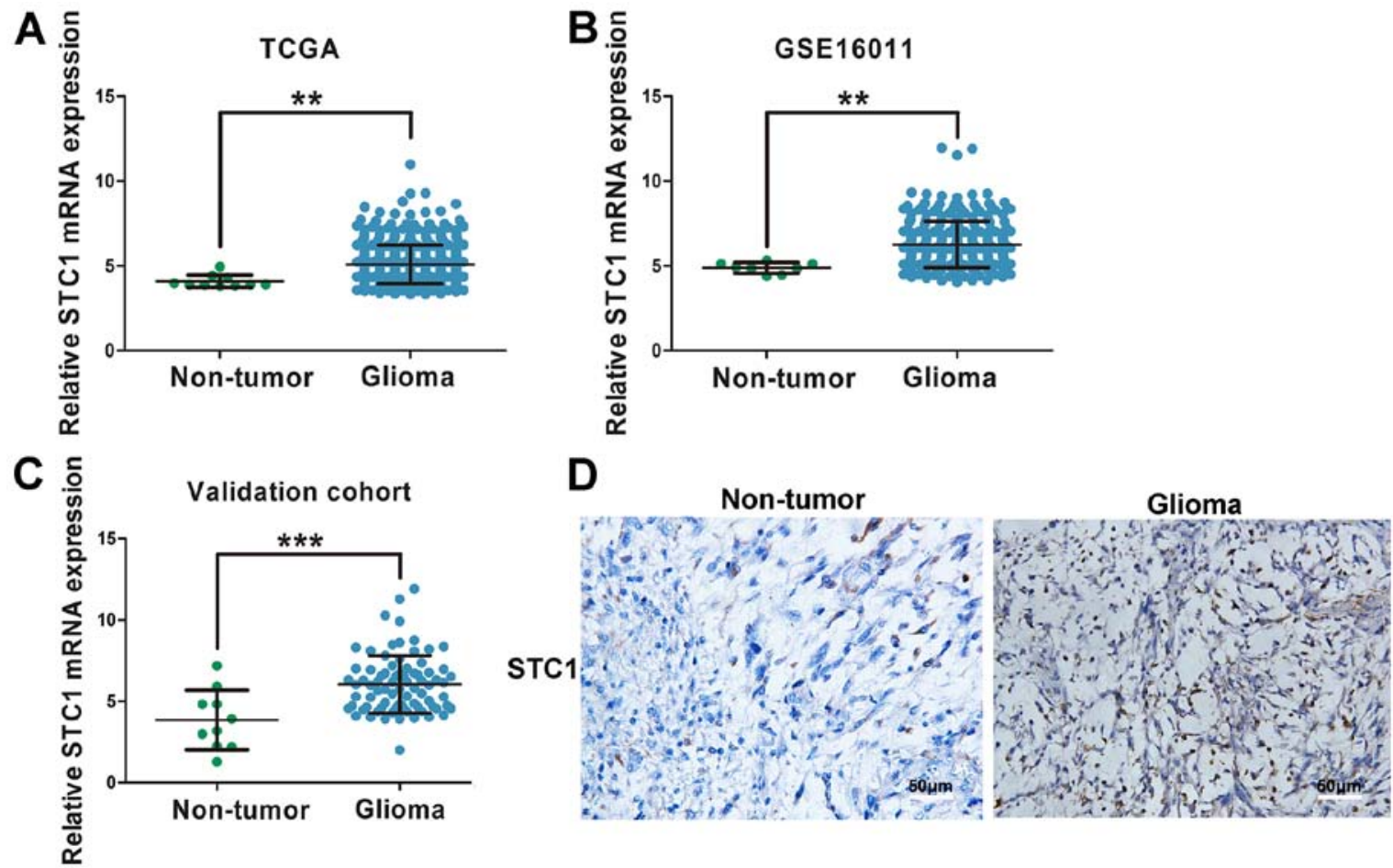

Figure 1. Expression of STC1 in non-tumor brain tissues and glioma tissues. STC1 expression in glioma tissues and non-tumor tissues from (A) TCGA and (B) GSE16011 datasets. (C) Reverse transcription-quantitative PCR was performed to detect STC1 mRNA expression in glioma tissues. (D) Representative images of STC1 expression in paraffin-embedded tissues. ${ }^{* *} \mathrm{P}<0.01,{ }^{* * * *} \mathrm{P}<0.001$ vs. non-tumor tissue. STC1, Stanniocalcin 1; TCGA, The Cancer Genome Atlas; CGGA, Chinese Glioma Genome Atlas.

and multivariate regression analyses were used to analyze the prognostic-related independent risk factors. $\mathrm{P}<0.05$ was considered to indicate a statistically significant different. All statistical analysis and graphics production were performed with SPSS v21 (IBM Corp.) and GraphPad Prism 5.0 software (GraphPad Software, Inc.).

\section{Results}

Expression of STC1 in glioma tissues. Normalized RNAseq gene expression from TCGA and GSE16011 datasets was used to analyze STC1 expression in glioma tissues. The results indicated that patients with glioma had higher expression levels of STC1 compared with non-tumor tissues (Fig. 1A and B). In order to validate this finding, RT-qPCR was performed on 80 glioma tissues and ten non-tumor brain tissues. The baseline characteristics of patients involved in the study are exhibited in Table I. It was demonstrated that STC1 mRNA expression was significantly elevated in glioma tissues compared with non-tumor tissues (Fig. 1C). In addition, IHC staining was performed, and it was identified that STC1 expression was higher in patients with glioma compared with those without brain tumor (Fig. 1D).

Association between expression of STC1 and glioma grade. It was demonstrated that the malignancy of glioma increased when the grade of tumor was increased The median overall survival of patients with glioblastoma [World Health Organization (WHO) IV] is 14 months and the 5-year survival rate is $<5 \%$ (22). In order to examine the correlation between STC1 expression and tumor grade, data from the four datasets,
TCGA, CGGA, GSE16011 and Rembrandt, were used. The results suggested that STC1 expression was elevated in patients with HGG compared with patients with LGG throughout the four datasets (Fig. 2A-D). Furthermore, in the validation cohort, compared with patients with LGG, there was an increased number of patients with high expression of STC1 in the HGG group according to the results of RT-qPCR (Fig. 2E) and IHC staining score (Fig. 2F and G).

Association between STC1 expression and glioma molecular subtypes. IDH status is an important molecular indicator for the prognosis of patients with glioma (23). Furthermore, it has been reported that the prognosis of patients with glioma with an IDH1 mutation is significantly improved compared with patients with IDH1 wild-type glioma. The results of the present study indicated that patients with IDH1 wild-type had a higher expression of STC1 compared with patients with the IDH1 mutation, according to TCGA, CGGA and GSE16011 datasets (Fig. 3A-C). This was not performed for the data obtained from the Rembrandt dataset due to the absence of IDH1/2 status information.

Moreover, differential expression among the four molecular subtypes was examined, and were classified based on transcriptome data $(24,25)$. In Rembrandt, STC1 expression was higher in the mesenchymal subtype compared with the neural subtype, but the difference was not significant (Fig. 3F). Furthermore, according to the TCGA and GSE16011 datasets, STC1 expression was highly expressed in mesenchymal glioma compared with other molecular subtypes (Fig. 3D and E), which has stronger invasive ability and increases resistance to chemotherapy compared with other subtypes (26). 
Table I. Baseline information of patients included in the study $(n=80)$.

\begin{tabular}{lc}
\hline Baseline information & Value \\
\hline Mean age \pm SD, years & $47.54 \pm 12.30$ \\
Sex, $\mathrm{n}$ & \\
Female & 51 \\
Male & 29 \\
Tumor location, $\mathrm{n}$ & \\
Supratentorial & 72 \\
Subtentorial & 8 \\
WHO grade, $\mathrm{n}$ & \\
I-II & 34 \\
III-IV & 46 \\
Mean follow-up time, months & 14.58 \\
\hline
\end{tabular}

WHO, World Health Organization.

Association between STC1 and prognosis in patients with glioma. The four datasets (TCGA, CGGA, Rembrandt and GSE16011) were used to investigate the prognostic role of STC1 in patients with glioma. Kaplan-Meier survival analyses results indicated that patients with high STC1 expression had a less favorable clinical prognosis compared with patients with low STC1 expression in all four datasets (Fig. 4A-D), which was also identified in the validation cohort. Furthermore, it was revealed that patients with glioma who expressed low levels of STC1 had a longer survival time compared with patients with high STC1 expression (Fig. 4E). To further determine the prognostic value of STC1 expression, multivariate Cox regression analysis was performed based on TCGA dataset. It was demonstrated that STC1 expression was significantly associated with the prognosis of patients with glioma via multivariate analysis [Hazard ratio $=0.52 ; \mathrm{P}<0.001$; Table II].

Role of STC1 in extracellular structure organization. According to mRNA sequencing in TCGA database, the expression of STC1 was divided into high and low groups to perform Gene Ontology analysis; the cut-off was $50 \%$ of STC1 expression. The results demonstrated that STC1 primarily enriched 'extracellular matrix organization' and 'extracellular structure organization', which indicates that STC1 may be closely associated with the migratory and invasive abilities of glioma (Fig. 5A). Only the results of biological function were presented to illustrate its potential oncogenic role in the present study.

To investigate the Gene Ontology results, association analysis between STC1 expression and various invasive-related protein expression was performed. It was found that STC1 expression was significantly associated with that of matrix metalloproteinase 2 (MMP2), MMP9, vimentin and Snail1 in TCGA database $(\mathrm{P}<0.0001 ;$ Fig. 5B-E).

\section{Discussion}

STC1 is a glycoprotein associated with calcium and phosphorus metabolism, but has rarely been studied in neurological diseases (27). Moreover, it was demonstrated that STC1 expression was significantly associated with malignancy, tumor grade, IDH status and subtype, in patients with glioma. Kaplan-Meier survival analyses also identified that patients with high STC1 expression levels had a less favorable clinical prognosis compared with patients with low STC1 expression, in both in silico analysis and cohort validation. Furthermore, biological process results of Gene Ontology analysis revealed that STC1 was primarily involved in 'extracellular structure organization'. It was also identified that STC1 expression in glioma was significantly correlated with MMP2, MMP9, vimentin and Snail1. Therefore, it was hypothesized that STC1 may represent a biomarker and therapeutic target in glioma.

A previous study reported that STC1 expression is elevated in lung adenocarcinoma and is positively correlated with tumor stage, using bioinformatics analysis and IHC staining validation (28). Moreover, STC1 is increased in patients with late recurrence breast cancer compared with patients with early recurrence, and its secretory form is associated with tumor size and disease-free survival $(13,29)$. Although STC1 has been widely studied in several cancer types, to the best of our knowledge, there are few studies on its effects in neurological diseases, especially glioma. In the present study, it was found that STC1 was upregulated in glioma tissues and its expression was enhanced as tumor grade increased, which indicates that STC1 may be an oncogene in glioma; which is in line with the previous reports $(14,15)$. Furthermore, elevated expression of STC1 is closely associated with the poor prognosis of patients with malignant tumors, such as gastric cancer (30), hepatocellular carcinoma (31) and esophageal squamous cell carcinoma (32).

It has been reported that STC1 may be a neuroprotectant in neurological diseases, and knockdown of STC1 expression in Amyloid $\beta$-treated human brain microvascular endothelial cells (HBMECs) increases the invasion of monocytes and apoptosis of HBMECs (33). However, further research is required to investigate the role of STC1 in cerebrovascular diseases. Using an ischemic mouse model, Durukan et al (34) revealed that STC1 was elevated under hypoxic condition and it was proven that STC1 was dispensable for functional recovery after ischemic stroke. Moreover, hypoxic conditions can induce the expression of STC1, and high expression of STC1 can enhance neuronal resistance to hypoxia (35). It has been demonstrated that hypoxic microenvironments are common in glioma tissues (35). In glioblastoma, tumor tissue hypoxia is an important indicator of malignancy of the tumor; the larger the hypoxic area, the higher the malignancy (35). Furthermore, a hypoxic environment can accelerate the proliferation, migration and invasiveness of tumor cells, and promote the malignant progression of glioma (36). Therefore, it was hypothesized that STC1 may regulated the malignant progression of glioma. To the best of our knowledge, the present study was the first to demonstrate that STC1 expression is elevated in glioma tissues compared with non-tumor brain tissues. In addition, the present results suggested that STC1 expression was significantly correlated with malignancy, as shown by tumor grade, IDH status and subtypes, of glioma, in both 

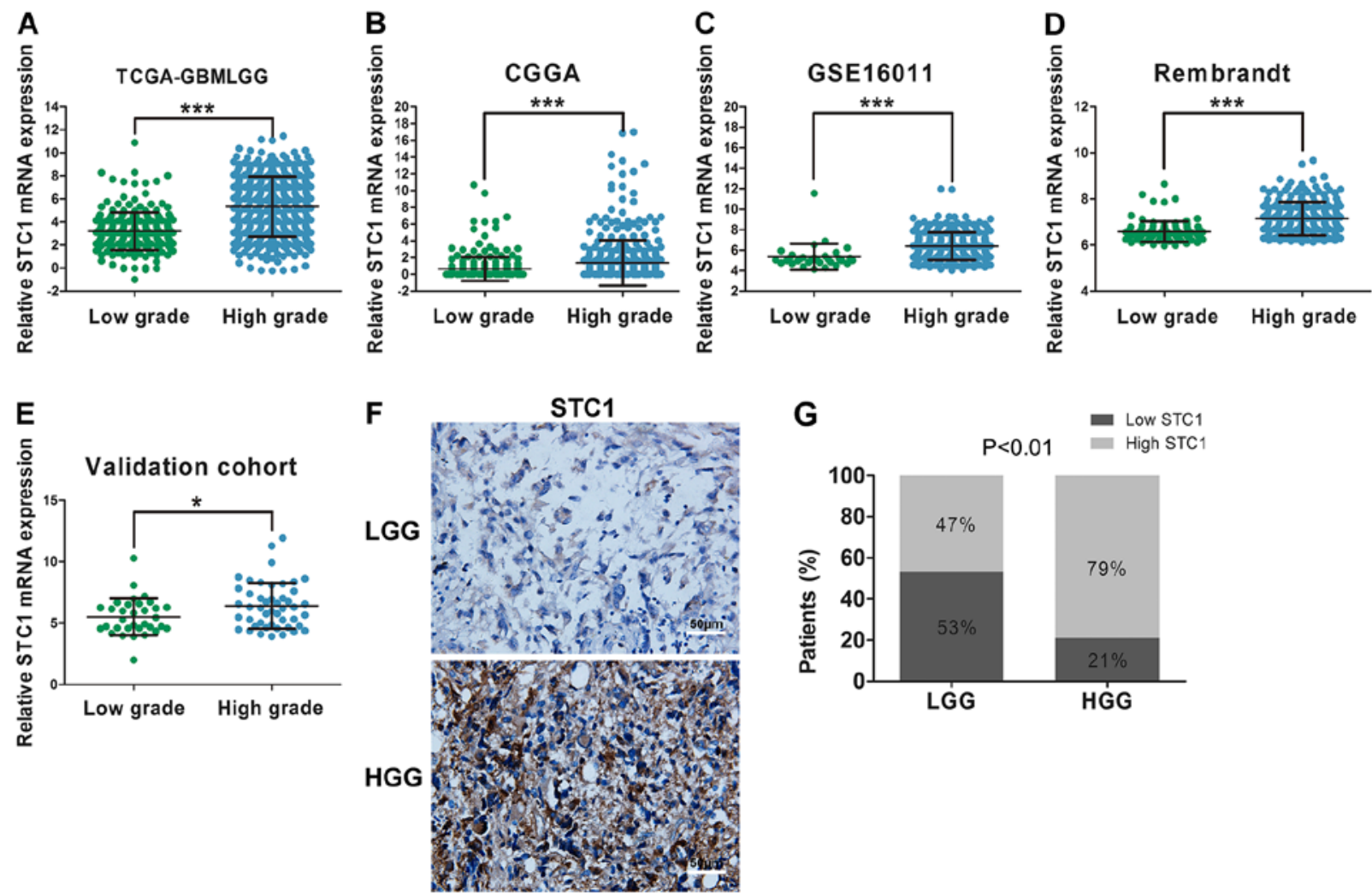

Figure 2. STC1 expression in different grades of glioma. Normalized mRNA expression of STC1 in HGG and LGG based on (A) TCGA, (B) CGGA, (C) GSE16011 and (D) Rembrandt. (E) Reverse transcription-quantitative PCR was performed to detect STC1 mRNA expression in glioma tissues. (F) Representative images of immunohistochemical staining of STC1 protein expression (G) in validation cohort. Fishers $\chi^{2}$ exact test was used to compare the difference between the two groups. Scale bar, $50 \mu \mathrm{m}$. ${ }^{*} \mathrm{P}<0.05,{ }^{* * * *} \mathrm{P}<0.001$ vs. LGG. STC1, Stanniocalcin 1; TCGA, The Cancer Genome Atlas; CGGA, Chinese Glioma Genome Atlas; LGG, low grade glioma; HGG, high grade glioma.

A
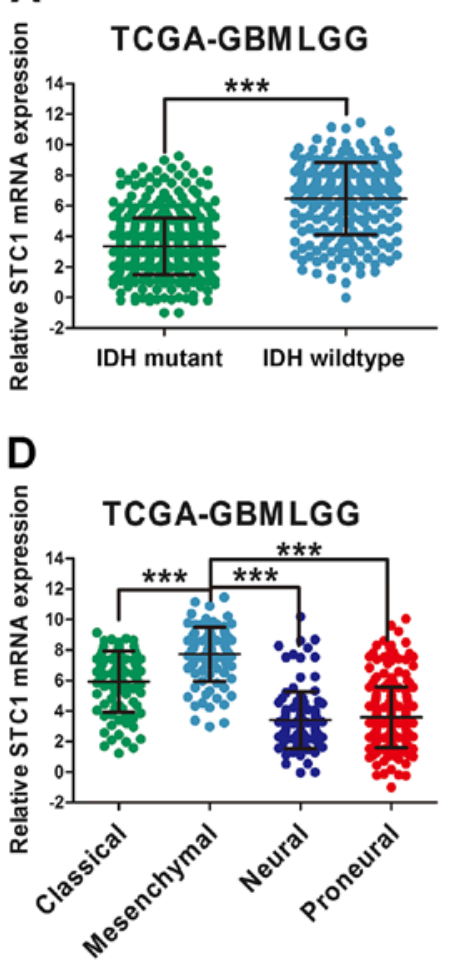

B

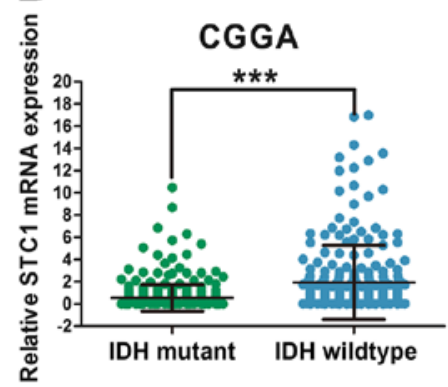

E

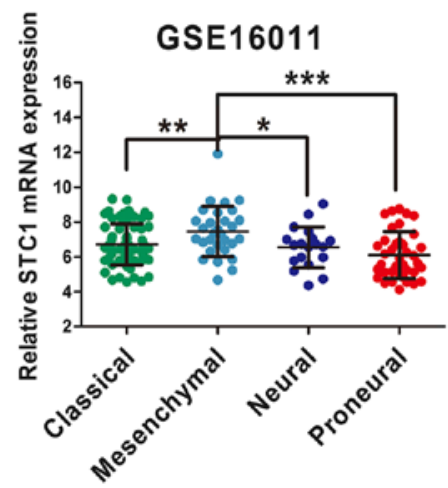

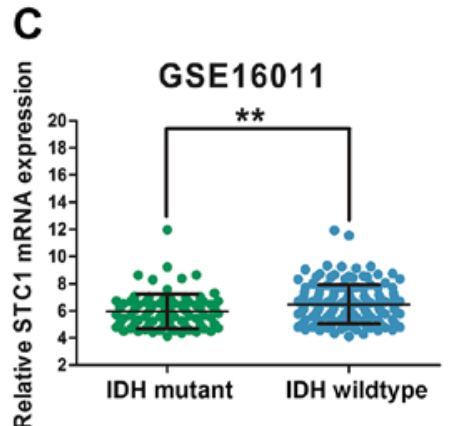

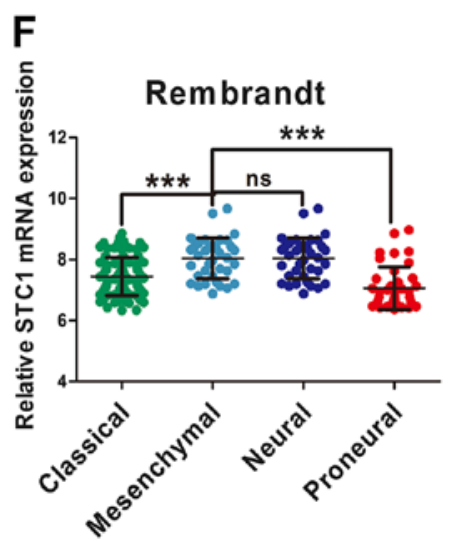

Figure 3. STC1 expression is associated with glioma molecular subtypes. Differential analysis of STC1 expression in patients with glioma with different IDH status according to (A) TCGA, (B) CGGA and (C) GSE16011 datasets. Expression of STC1 in different glioma molecular subtypes according to (D) TCGA, (E) GSE16011 and (F) Rembrandt datasets. ${ }^{*} \mathrm{P}<0.05,{ }^{* *} \mathrm{P}<0.01,{ }^{* * * *} \mathrm{P}<0.001$. ns, no significance; STC1, Stanniocalcin 1; TCGA, The Cancer Genome Atlas; CGGA, Chinese Glioma Genome Atlas; IDH, isocitrate dehydrogenase; GBMLGG, Glioblastoma and low grade glioma. 
Table II. Univariate analysis and multivariate Cox analysis of clinical prognostic parameters in The Cancer Genome Atlas.

\begin{tabular}{|c|c|c|c|c|}
\hline \multirow[b]{2}{*}{ Variable } & \multicolumn{2}{|c|}{ Univariate cox regression } & \multicolumn{2}{|c|}{ Multivariate cox regression } \\
\hline & $\mathrm{HR}(95 \% \mathrm{CI})$ & P-value & HR $(95 \%$ CI $)$ & P-value \\
\hline $\begin{array}{l}\text { Age, years } \\
\geq 60 \text { vs. }<60\end{array}$ & $0.31(0.23-0.41)$ & $<0.001$ & $1.10(0.76-1.59)$ & 0.63 \\
\hline $\begin{array}{l}\text { Sex } \\
\text { Female vs. male }\end{array}$ & $1.14(0.87-1.49)$ & 0.36 & - & - \\
\hline $\begin{array}{l}\text { WHO Grade } \\
\text { High vs. low }\end{array}$ & $0.18(0.12-0.27)$ & $<0.001$ & $0.44(0.29-0.68)$ & $<0.001$ \\
\hline $\begin{array}{l}\text { Isocitrate dehydrogenase } 1 \text { status } \\
\text { Wild-type vs. mutant }\end{array}$ & $0.10(0.07-0.13)$ & $<0.001$ & $0.18(0.12-0.27)$ & $<0.001$ \\
\hline $\begin{array}{l}\text { MGMT promoter } \\
\text { Unmethylated vs. methylated }\end{array}$ & $0.37(0.28-0.50)$ & $<0.001$ & $0.99(0.72-1.36)$ & 0.95 \\
\hline $\begin{array}{l}\text { Molecular subtype } \\
\text { ME vs. others }\end{array}$ & $0.22(0.17-0.30)$ & $<0.001$ & $0.57(0.41-0.80)$ & 0.001 \\
\hline $\begin{array}{l}\text { Stanniocalcin } 1 \text { expression } \\
\text { High vs. low }\end{array}$ & $0.20(0.15-0.26)$ & $<0.001$ & $0.52(0.38-0.71)$ & $<0.001$ \\
\hline
\end{tabular}

MGMT, $\mathrm{O}^{6}$-methylguanine-DNA methyltransferase; WHO, World Health Organization; ME, mesenchymal, comparison with other molecular subtypes ; HR, hazard ratio; CI, confidence interval.

A

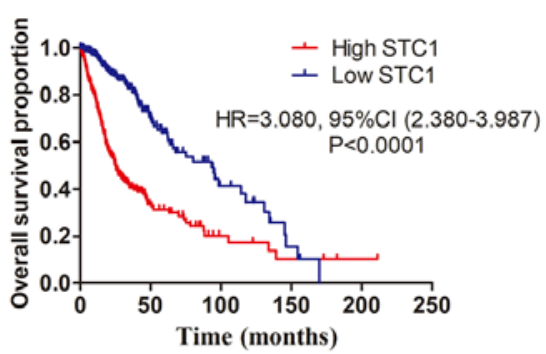

TCGA

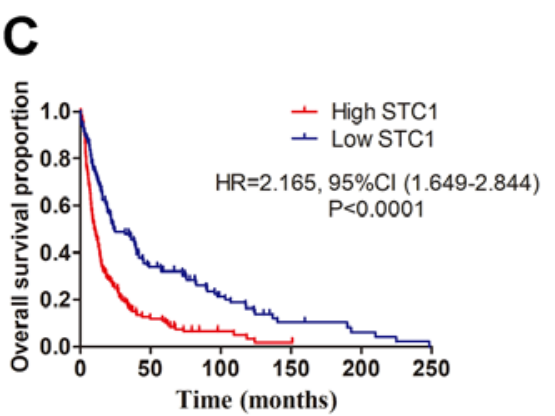

GSE16011
B

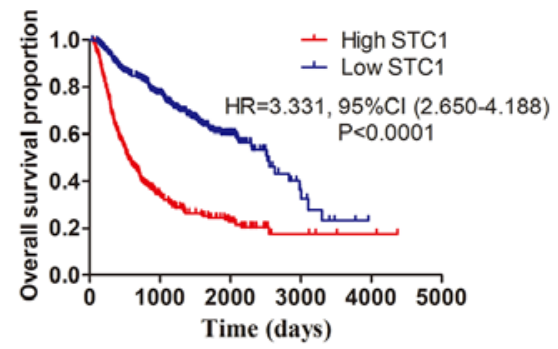

CGGA

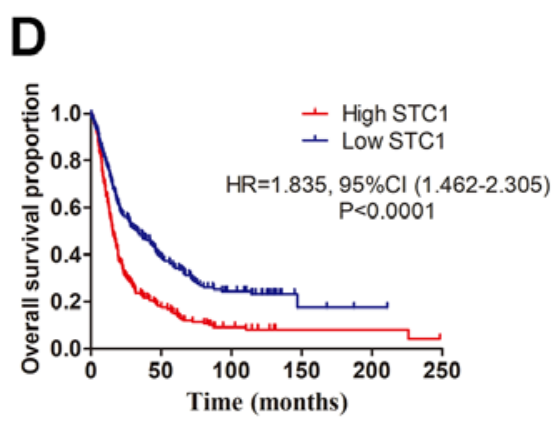

Rembrandt

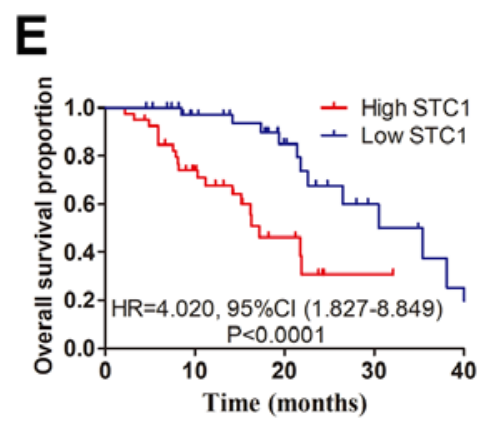

Validation cohort

Figure 4. STC1 expression is associated with prognosis in patients with glioma. Association between STC1 expression and prognosis of glioma in (A) TCGA, (B) CGGA, (C) GSE16011 and (D) Rembrandt datasets was analyzed. (E) Validation cohort was used to assess the prognostic role of STC1 in patients with glioma. HR, hazard ratio; STC1, Stanniocalcin 1; TCGA, The Cancer Genome Atlas; CGGA, Chinese Glioma Genome Atlas.

in silico analysis and in the validation cohort. Collectively, the present results indicated the potential oncogenic role of STC1 in the progression of glioma. Furthermore, higher expression of STC1 in glioma tissues was associated with poorer prognosis of overall survival, which demonstrated that STC1 may be a biomarker in patients with glioma. 
A
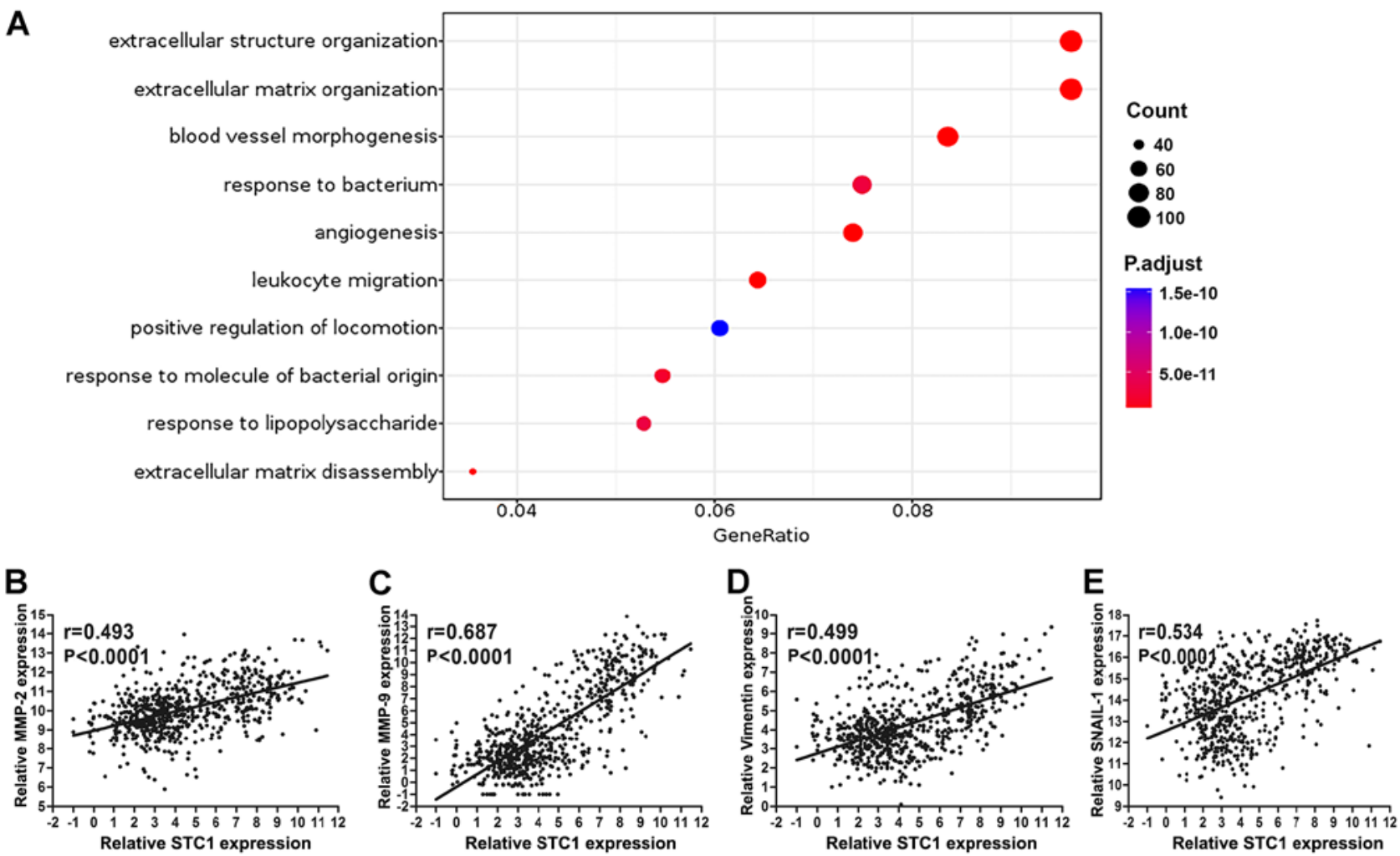

Figure 5. STC1 involved in extracellular structure organization. (A) Biological process of Gene Ontology analysis of STC1 based on TCGA dataset. Correlation between STC1 and invasion-related markers, including (B) MMP2, (C) MMP9, (D) Snail1 and (E) vimentin from the TCGA dataset. STC1, Stanniocalcin 1; TCGA, The Cancer Genome Atlas; CGGA; MMP, matrix metalloproteinase.

STC1 has been reported to serve as an oncogene. For example, Li et al (15) reported that STC1 expression is upregulated in glioma stem cells, and it directly binds NOTCH1, which subsequently mediates the stem-like traits of glioma cells. Moreover, hypoxia induces the expression of STC1 in the tumor microenvironment, thus indicating that STC1 may be a crucial factor mediating cancer metastasis and chemoresistance (37). In addition, Wang et al (30) revealed that STC1 expression promotes gastric cancer cell proliferation, migration and invasion under hypoxia. Furthermore, STC1 also exhibits the ability to enhance tumor growth and reprogrammed metabolism in hepatocellular carcinoma (13). A previous study has also reported hypoxia as an invasion-promoting factor in glioma cells (38). In the present study, biological process of Gene Ontology analysis results demonstrated that STC1 was mainly involved in the 'extracellular matrix organization', which suggested that STC1 may be closely associated with the migration and invasion abilities of glioma cells. It has been shown that infiltration of glioma cells into surrounding non-tumor brain tissue is an important process in promoting malignancy of the tumor (38). Therefore, targeted interventions to inhibit the invasion of glioma cells may represent an important strategy for the treatment of glioma.

The present study had several limitations. The present results suggest that STC1 expression is elevated in glioma tissues compared with non-tumor brain tissues. While expression of STC1 in non-tumor brain tissues may be partly attributable to the effects of traumatic brain injury (TBI) or hypertensive intracerebral haemorrhage, which was not controlled for in present study. In addition, the sample size in the validation cohort was small and a larger sample size is required for future validation. Finally, only $\mathrm{GO}$ analysis was performed, future in vitro and in vivo studies are required to validate the functional role of STC1 in glioma.

In conclusion, STC1 expression was upregulated in glioma tissues and was significantly associated with tumor grade and molecular characteristics, in both in silico analysis and cohort validation. Furthermore, it was demonstrated that patients with higher STC1 expression had shorter overall survival times compared with those with lower STC1 expression. Gene Ontology results also suggested that STC1 may be a key regulator of invasiveness in gliomas. Therefore, the present results indicated that STC1 may represent a novel biomarker and a potential target for the treatment of glioma.

\section{Acknowledgements}

Not applicable.

\section{Funding}

No funding was received.

\section{Availability of data and materials}

The datasets used and/or analyzed during the present study are available from the corresponding author on reasonable request. 


\section{Authors' contributions}

WJL and DC designed the present study. WJL, DC and HW acquired and analyzed the data. JLH and WJL drafted the initial manuscript and made revisions for important intellectual content. JLH also designed the study and was responsible for analysis and interpretation of data. JLH agreed to be accountable for all aspects of the work in ensuring that questions related to the accuracy or integrity of any part of the work are appropriately investigated and resolved. All authors read and approved the final manuscript.

\section{Ethics approval and consent to participate}

This study was approved by the Ethics Committee of Shenzhen People's Hospital and informed consent of every patient included was signed by their relatives.

\section{Patient consent for publication}

Not applicable.

\section{Competing interests}

The authors declare that they have no competing interests.

\section{References}

1. Ostrom QT, Gittleman H, Truitt G, Boscia A, Kruchko C and Barnholtz-Sloan JS: CBTRUS statistical report: Primary brain and other central nervous system tumors diagnosed in the United States in 2011-2015. Neuro Oncol 20 (Suppl 4): iv1-iv86, 2018.

2. Albain KS, Swann RS, Rusch VW, Turrisi AT III, Shepherd FA, Smith C, Chen Y, Livingston RB, Feins RH, Gandara DR, et al: Radiotherapy plus chemotherapy with or without surgical resection for stage III non-small-cell lung cancer: A phase III randomised controlled trial. Lancet 374: 379-386, 2009.

3. Ostrom QT, Cote DJ, Ascha M, Kruchko C and Barnholtz-Sloan JS: Adult glioma incidence and survival by race or ethnicity in the United States from 2000 to 2014. JAMA Oncol 4: 1254-1262, 2018.

4. Bralten LB and French PJ: Genetic alterations in glioma. Cancers (Basel) 3: 1129-1140, 2011.

5. Ohgaki H, Dessen P, Jourde B, Horstmann S, Nishikawa T, Di Patre PL, Burkhard C, Schüler D, Probst-Hensch NM, Maiorka PC, et al: Genetic pathways to glioblastoma: A population-based study. Cancer Res 64: 6892-6899, 2004.

6. Zheng H, Ying H, Yan H, Kimmelman AC, Hiller DJ, Chen AJ, Perry SR, Tonon G, Chu GC, Ding Z, et al: p53 and Pten control neural and glioma stem/progenitor cell renewal and differentiation. Nature 455: 1129-1133, 2008.

7. Chou MY, Lin CH, Chao PL, Hung JC, Cruz SA and Hwang PP: Stanniocalcin-1 controls ion regulation functions of ion-transporting epithelium other than calcium balance. Int J Biol Sci 11: 122-132, 2015.

8. Hayase S, Sasaki Y, Matsubara T, Seo D, Miyakoshi M, Murata T, Ozaki T, Kakudo K, Kumamoto K, Ylaya K, et al: Expression of stanniocalcin 1 in thyroid side population cells and thyroid cancer cells. Thyroid 25: 425-436, 2015.

9. Zhou H, Li YY, Zhang WQ, Lin D, Zhang WM and Dong WD: Expression of stanniocalcin-1 and stanniocalcin-2 in laryngeal squamous cell carcinoma and correlations with clinical and pathological parameters. PLoS One 9: e95466, 2014.

10. Song H, Xu B and Yi J: Clinical significance of stanniocalcin-1 detected in peripheral blood and bone marrow of esophageal squamous cell carcinoma patients. J Exp Clin Cancer Res 31: 35, 2012.

11. Du YZ, Gu XH, Li L and Gao F: The diagnostic value of circulating stanniocalcin-1 mRNA in non-small cell lung cancer. J Surg Oncol 104: 836-840, 2011.

12. Liu G, Yang G, Chang B, Mercado-Uribe I, Huang M, Zheng J, Bast RC, Lin SH and Liu J: Stanniocalcin 1 and ovarian tumorigenesis. J Natl Cancer Inst 102: 812-827, 2010.
13. Chan KK, Leung CO, Wong CC, Ho DW, Chok KS, Lai CL, $\mathrm{Ng}$ IO and Lo RC: Secretory stanniocalcin 1 promotes metastasis of hepatocellular carcinoma through activation of JNK signaling pathway. Cancer Lett 403: 330-338, 2017.

14. Su J, Guo B, Zhang T, Wang K, Li X and Liang G: Stanniocalcin-1, a new biomarker of glioma progression, is associated with prognosis of patients. Tumour Biol 36: 6333-6339, 2015.

15. Li Y, He ZC, Zhang XN, Liu Q, Chen C, Zhu Z, Chen Q, Shi Y, Yao XH, Cui YH, et al: Stanniocalcin-1 augments stem-like traits of glioblastoma cells through binding and activating NOTCH1. Cancer Lett 416: 66-74, 2018.

16. Bowman RL, Wang Q, Carro A, Verhaak RG and Squatrito M: GlioVis data portal for visualization and analysis of brain tumor expression datasets. Neuro Oncol 19: 139-141, 2017.

17. Gravendeel LA, Kouwenhoven MC, Gevaert O, de Rooi JJ, Stubbs AP, Duijm JE, Daemen A, Bleeker FE, Bralten LB, Kloosterhof NK, et al: Intrinsic gene expression profiles of gliomas are a better predictor of survival than histology. Cancer Res 69: 9065-9072, 2009.

18. Hu H, Mu Q, Bao Z, Chen Y, Liu Y, Chen J, Wang K, Wang Z, Nam Y, Jiang B, et al: Mutational landscape of secondary glioblastoma guides MET-targeted trial in brain tumor. Cell 175: 1665-1678.e18, 2018.

19. Louis DN, Perry A, Reifenberger G, von Deimling A, Figarella-Branger D, Cavenee WK, Ohgaki H, Wiestler OD, Kleihues P and Ellison DW: The 2016 world health organization classification of tumors of the central nervous system: A summary. Acta Neuropathol 131: 803-820, 2016.

20. Verhaak RG, Hoadley KA, Purdom E, Wang V, Qi Y, Wilkerson MD, Miller CR, Ding L, Golub T, Mesirov JP, et al: Integrated genomic analysis identifies clinically relevant subtypes of glioblastoma characterized by abnormalities in PDGFRA, IDH1, EGFR, and NF1. Cancer Cell 17: 98-110, 2010.

21. Livak KJ and Schmittgen TD: Analysis of relative gene expression data using real-time quantitative PCR and the 2(-Delta Delta C(T)) method. Methods 25: 402-408, 2001.

22. Stupp R, Mason WP, Van Den Bent MJ, Weller M, Fisher B, Taphoorn MJ, Belanger K, Brandes AA, Marosi C, Bogdahn U, et al: Radiotherapy plus concomitant and adjuvant temozolomide for glioblastoma. N Engl J Med 352: 987-996, 2005.

23. Pekmezci M, Rice T, Molinaro AM, Walsh KM, Decker PA, Hansen H, Sicotte H, Kollmeyer TM, McCoy LS, Sarkar G, et al: Adult infiltrating gliomas with WHO 2016 integrated diagnosis: Additional prognostic roles of ATRX and TERT. Acta Neuropathol 133: 1001-1016, 2017.

24. Shen R, Mo Q, Schultz N, Seshan VE, Olshen AB, Huse J, Ladanyi $M$ and Sander C: Integrative subtype discovery in glioblastoma using icluster. PLoS One 7: e35236, 2012.

25. Morokoff A, Ng W, Gogos A and Kaye AH: Molecular subtypes, stem cells and heterogeneity: Implications for personalised therapy in glioma. J Clin Neurosci 22: 1219-1226, 2015.

26. Ramirez YP, Weatherbee JL, Wheelhouse RT and Ross AH: Glioblastoma multiforme therapy and mechanisms of resistance. Pharmaceuticals (Basel) 6: 1475-1506, 2013.

27. Kikuchi M, Nakano Y, Nambo Y, Haneda S, Matsui M, Miyake Y, Macleod JN, Nagaoka K and Imakawa K: Production of calcium maintenance factor stanniocalcin-1 (STC1) by the equine endometrium during the early pregnant period. J Reprod Dev 57: 203-211, 2011.

28. Du YZ, Gu XH, Cheng SF, Li L, Liu H, Hu LP and Gao F: The oncogenetic role of stanniocalcin 1 in lung adenocarcinoma: A promising serum candidate biomarker for tracking lung adenocarcinoma progression. Tumour Biol 37: 5633-5644, 2016.

29. Brantley KD, Kjaersgaard A, Cronin-Fenton D, Yacoub R, Nielsen AS, Lauridsen KL, Hamilton-Dutoit S and Lash TL: Stanniocalcin expression as a predictor of late breast cancer recurrence. Cancer Epidemiol Biomarkers Prev 27: 653-659, 2018.

30. Wang Y, Qi Z, Zhou M, Yang W, Hu R, Li G, Ma X and Zhang Z: Stanniocalcin 1 promotes cell proliferation, chemoresistance and metastasis in hypoxic gastric cancer cells via Bcl2. Oncol Rep 41: 1998-2008, 2019.

31. Leung CC and Wong CK: Effects of STC1 overexpression on tumorigenicity and metabolism of hepatocellular carcinoma. Oncotarget 9: 6852-6861, 2018.

32. Shirakawa M, Fujiwara Y, Sugita Y, Moon JH, Takiguchi S, Nakajima K, Miyata H, Yamasaki M, Mori M and Doki Y: Assessment of stanniocalcin-1 as a prognostic marker in human esophageal squamous cell carcinoma. Oncol Rep 27: 940-946, 2012. 
33. Li K, Dong D, Yao L, Dai D, Gu X and Guo L: Identification of STC1 as an beta-amyloid activated gene in human brain microvascular endothelial cells using cDNA microarray. Biochem Biophys Res Commun 376: 399-403, 2008.

34. Durukan Tolvanen A, Westberg JA, Serlachius M, Chang AC, Reddel RR, Andersson LC and Tatlisumak T: Stanniocalcin 1 is important for poststroke functionality, but dispensable for ischemic tolerance. Neuroscience 229: 49-54, 2013.

35. Ito Y, Zemans R, Correll K, Yang IV, Ahmad A, Gao B and Mason RJ: Stanniocalcin-1 is induced by hypoxia inducible factor in rat alveolar epithelial cells. Biochem Biophys Res Commun 452: 1091-1097, 2014.

36. Monteiro AR, Hill R, Pilkington GJ and Madureira PA: The Role of Hypoxia in Glioblastoma Invasion. Cells 6: E45, 2017.
37. Yeung HY, Lai KP, Chan HY, Mak NK, Wagner GF and Wong CK: Hypoxia-inducible factor-1-mediated activation of stanniocalcin-1 in human cancer cells. Endocrinology 146: 4951-4960, 2005.

38. Jensen RL: Brain tumor hypoxia: Tumorigenesis, angiogenesis, imaging, pseudoprogression, and as a therapeutic target. J Neurooncol 92: 317-335, 2009.

(c) (i) () $($ This work is licensed under a Creative Commons EY NO ND Attribution-NonCommercial-NoDerivatives 4.0 International (CC BY-NC-ND 4.0) License. 